\title{
Empirical Analysis of Body Constitution and Food Intake in Persons with Type 2 Diabetes from a TCM Perspective
}

\author{
Peggy Yee-Chi Wong ${ }^{1, *}$, Samantha Mei-Che Pang ${ }^{2}$ and Rose Yuk-Pui Chan ${ }^{2}$ \\ ${ }^{1}$ Dietetic Department, Tuen Mun Hospital, Tuen Mun, New Territories West Cluster, Hong Kong \\ ${ }^{2}$ Hong Kong Polytechnic University, Hong Kong
}

\begin{abstract}
This study examined the correlations between body constitution (BC) and food intake in a sample of persons with Type 2 Diabetes (T2DM) from a perspective of traditional Chinese medicine (TCM). Past research on BC of persons with diabetes (DM) from a TCM perspective revealed imbalanced state of Yin and Yang in terms of Yin-deficiency (YID), Yang-deficiency (YAD), and Yin-Yang-deficiency (YYD). However, no studies have attempted to find out if daily food intake has an influence on Yin-Yang balance. The present study adapted a mixed method, which constituted of two phases. Phase one involved an exploratory case study $(n=18)$ conducted between May and June 2011 and phase two, a descriptive correlation study $(n=210)$ between October and December 2013. Results showed that in phase-one, three cases showed YID and higher food intake in hot/warm nature, 12 cases with YAD and higher food intake in cold/cool nature while three cases with Yin-Yang-deficiency (YYD) and extremely high food intake in cold/cool nature. In phasetwo, Spearman's correlation coefficient between food intake and YID presentations (YIDPs) (hot/warm food: rho $=0.34$, $p=0.000$; cold/cool food: rho=0.18, $p=0.006$ ); YAD presentations (YADPs) (hot/warm food: rho $=0.18, p=0.008$; cold $/ \mathrm{cool}$ food: rho=0.2, $p=0.006$ ); and YYD presentations (YYDPs) (hot/warm food: rho $=0.29, p=0.006$; cold/cool food: rho $=0.2$, $p=0.003$ ) have been noted. The findings concluded that persons with T2DM and YIDPS, YADPs, or YYDPs tend to have food intakes higher in hot/warm nature or cold/cool nature.
\end{abstract}

Keywords: Type 2 Diabetes, Yin-Yang theory, Yin-Yang interaction, body constitution, Yin-deficiency, Yangdeficiency, Yin-Yang-deficiency, TCM food properties, mixed method study.

\section{INTRODUCTION}

Diabetes mellitus (DM) prevalence has been increasing [1] and DM remains to be persistent public health burden worldwide [2]. Conventional dietary therapy (CDT) helps stabilize blood glucose level with regard to nutrition component. However, it is not uncommon for a dietician to encounter questions from some Chinese persons with DM who are interested in knowing about specific food types that would induce an adverse reaction by reflecting cold or hot presentations. Common reactions are "fruit is too cold/cool for me"; "chicken and beef are too hot/warm for me", "raw vegetables can cause my tummy a cold-dampness leading to diarrhoea"; "my body would become a heatdryness if I eat baked foods"; and "brined vegetable in soup helps clear my inner heat". From the perspective of traditional Chinese medicine (TCM), the mentioned "cold/cool" and "hot/warm"; and "cold-dampness" and "heat-dryness" are common terms that describe the natures of food [3] and body constitution Presentations (BCPs) respectively [4]. In view of the interests in TCM food natures by persons with DM, we surveyed 112 persons with DM between September and November 2009 in the New Territories West Cluster of Hong Kong. $69(61.9 \%)$ of the survey participants believed

${ }^{*}$ Address correspondence to this author at the Dietetic Department, Tuen Mun Hospital, Tuen Mun, New Territories West Cluster, Hong Kong; Tel: (852)-24685108; Fax: (852)-24631314; E-mail: wongp@ha.org.hk

E-ISSN: $1929-5634 / 14$ that food had influence on their body constitution (BC) apart from blood glucose stabilization. According to two TCM scholars who are renowned for their contributions to the study of $B C$, definition of $B C$ can be summarized as the special state of a population or an individual exhibiting a stable state of physical structure along with stable physiological, metabolic and psychological functions formed during growth, development and aging under the influence of geography and environment as well as individual heredity [5-6]. BC represents the natures of the person's morphosis, physical, physiological, psychological, and metabolic components at one point of time, or sometimes a short period [7-8]. It is congenital in an individual body and is, however, also affected by acquired factors at a slow rate throughout the growth period and aging in life such as food intake [9], emotion [10], age [11], environmental and geographical influences [12], and disease [13]. In fact, BC is perceived as a balanced state of Yin and Yang from the perspective of TCM. Underpinned TCM is the Yin-Yang theory.

Yin-Yang theory is the foundation of TCM [14]. Yin and Yang play an important role in the clinical diagnosis and treatment. It is said in the first medical text in China, the Yellow Emperor's Canon of Internal Medicine: "He who is good at diagnosis always observes the complexion of the patient and palpates the pulse first, to distinguish whether the disease belongs to Yin or Yang." [15]. Yin characterizes the 
cold-dampness quality of nature while Yang characterizes the heat-dryness of nature [16]. In human physiology, Yin is the vital essence inside the body corresponds to blood, body fluid (sweat, tear, saliva, and drool), nutrients [17], and other vital substances, such as the hormone, enzyme, trace chemical elements and nerve matters [18] while Yang corresponds to vital energy utilizing the vital essence [17]. A balance of Yin and Yang leads to a gentle BC (healthy $\mathrm{BC}$ ), thus contributing to the prevention of disease occurrence and progression. By interaction of Yin and Yang, disease occurs in deviated BC (unhealthy BC) when either Yin or Yang is in excess or deficiency-opposition of Yin and Yang [17]; an extreme deficiency of either Yin or Yang causes the weakening of both Yin and Yang-interdependence between Yin and Yang [17] while an extreme excess of either causes a transformation of Yang or Yin-mutual transformation between Yin and Yang [17].

Common types of BC in DM are Yin-deficiency (YID), Yang-deficiency (YAD), and Yin-Yang-deficiency (YYD) [18-19]. DM occurs when inner heat-dryness is induced by an intake of food that is higher in Yang-hot nature [20], emotion [21], exhaustion [22], and congenital weak organs [15]. Heat-dryness is a reflection of YID type of BC [23]. YID of any one organ extends its heat-dryness to other organs causing extended organ dysfunction. For instance, SPLEEN/STOMACH heat leads to the failure of ascending water to the LUNG causing 'thirst' and descending nutrients to the small intestine causing 'emaciation' [24]. KIDNEY YID fails to distribute the essence and water up to the LUNG and SPLEEN/STOMACH causing 'thirst' and 'emaciation' respectively [24]. So, YID is the initial phase of DM [25]. It can further change to YAD or YYD types of BC if not regulated to return a balance state of Yin and Yang [26].

BC can be regulated by a balance of Yin and Yang with food nature from the perspective of TCM [27]. In the theory of TCM, food is categorized as having different natures [28]. They are cold, cool, warm, hot, and neutral natures [29]. Food of cold/cool nature is suitable for YID type of BC because it nourishes Yin by lowering the inner "fire" [28]. Likewise, food of hot/warm nature is suitable for YAD type of $B C$ because it strengthens Yang by dispelling the inner 'cold' [28]. In contrast, food intake higher in cold/cool nature causes $Y A D$ type of $B C$ while that of hot/warm nature causes YID type of BC. One more type of food nature is neither cold/cool nor hot/warm. It is called "neutral" or "even".
This lies between the warm and the cool natures and does not have an influence on either YID or YAD type of $\mathrm{BC}[30]$.

Empirical studies found that deviated $\mathrm{BC}$ can be regulated by food means in TCM [31-32]. Shen et al. [28] reported that hypertensive participants with YID dropped in numbers in the intervention group after four, eight, 12 and 16 weeks follow-up $(n=9,12,25,18$, $2=46.08, p<0.001$, using Friedman test), while the numbers in the control group did not significantly change $(n=0,2,1,3,2=6.8, p<0.147)$. The results of this randomized clinical study supports that food of cold/cool nature was effective in YID regulation, improving quality of life (QoL), and it controlled blood pressure. However, to our knowledge, no studies have attempted to find out if daily food intake has an influence on BCP. Since T2DM afflicts a majority of DM population [33], we, therefore, designed a mixed method study to explore the correlations between BCPs and daily food intake in persons with T2DM.

\section{OBJECTIVES AND PROPOSITION}

The objectives of the present study are to (1) assess the BCPs in a sample of persons with T2DM from a TCM perspective (TCMP); (2) identify the foods consumed according to the TCM food natures in a sample of persons with T2DM; and (3) examine the correlations between BCPs and TCM food natures in a sample of persons with T2DM. In accordance with the theory of Yin-Yang interaction, the present study proposes the following:

Persons with T2DM and YIDPs, YADPs, or YYDPs have food intakes higher in hot/warm nature or cold/cool nature from the perspective of TCM.

\section{METHODOLOGY}

\section{Design}

This study adapted a two-phase mixed method design [34] including case study [35] and descriptive correlation study [36]. Figure 1 summarizes the study design. This study had obtained ethical approval from the Human Subjects Ethics Sub-committee of the Hong Kong Polytechnic University prior to the first stage of research which was a case study.

\section{Case Study}

In phase one, a sample of 18 persons with T2DM who met the inclusion criteria was firstly selected from 


\section{Study schedule of mixed method study}

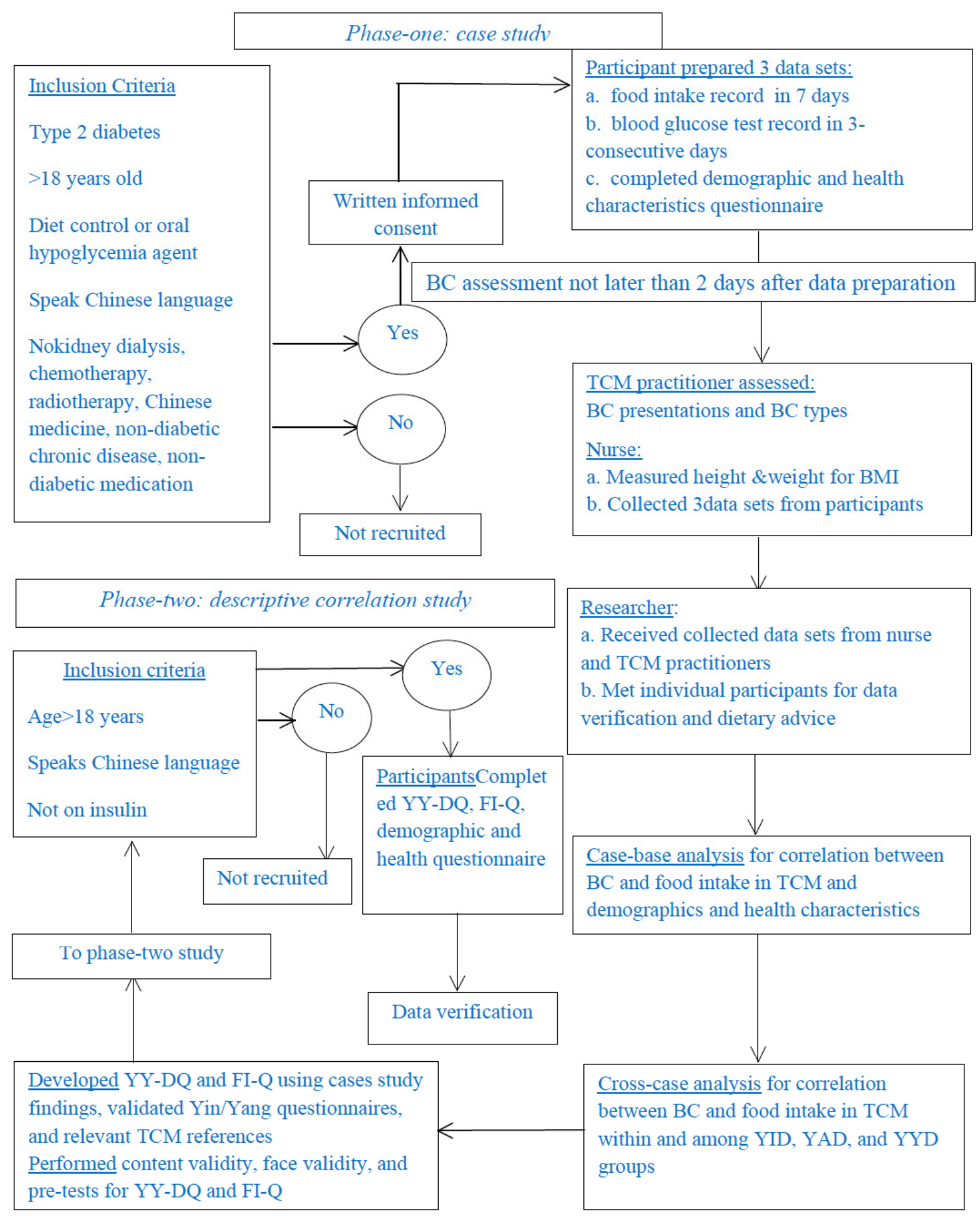

$\mathrm{BC}=$ body constitution; $\mathrm{YID}=\mathrm{Y}$ in-deficiency; $\mathrm{YAD}=\mathrm{Y}$ ang-deficiency; $\mathrm{Y} Y \mathrm{D}=\mathrm{Y}$ in-Yang-deficiency; $\mathrm{YY}-\mathrm{DQ}=\mathrm{Y}$ in-Yangdeficiency Questionnaire; FI-Q=Food Frequency Questionnaire; BMI=body mass index

Figure 1: Study schedule of mixed method study.

a non-profit association for persons with DM. They all signed a consent form to participate in the study. The criteria for selection included those who were (1) aged over 18 years, (2) diagnosed to be diabetic for $n$ number of years, (3) on diet control or on oral diabetic medications, (4) able to speak Chinese language, and 
Table 1: The Yin/Yang-deficiency Questionnaire (YY-DQ)

\begin{tabular}{|c|c|c|c|c|c|}
\hline Your current presentations & \multicolumn{5}{|c|}{ Put a" $\checkmark "$ if applicable } \\
\hline \multicolumn{6}{|l|}{ Body Surface } \\
\hline \multicolumn{6}{|c|}{ I wear thin clothes due to the hot temperature } \\
\hline \multicolumn{6}{|l|}{ I feel hot and cold on and off } \\
\hline \multicolumn{6}{|c|}{ I wear thick clothes due to fear of the cold temperature } \\
\hline \multicolumn{6}{|c|}{ I feel uncomfortable with wind blowing } \\
\hline \multicolumn{6}{|c|}{ I have hot flushes at night } \\
\hline \multicolumn{6}{|c|}{$\begin{array}{l}\text { I have heavy sweating but not because of the hot temperature/intensive } \\
\text { exercise }\end{array}$} \\
\hline \multicolumn{6}{|l|}{ Four Limbs } \\
\hline \multicolumn{6}{|l|}{ I feel hot in my palm and arch } \\
\hline \multicolumn{6}{|c|}{$\begin{array}{l}\text { I have pain in my knee, loin, shoulder, and back } \\
\text { (feeling better with heat application) }\end{array}$} \\
\hline \multicolumn{6}{|l|}{ Head and Neck } \\
\hline \multicolumn{6}{|c|}{ I have hot flushes in my face especially during the afternoon time } \\
\hline \multicolumn{6}{|c|}{ My face is red hot particularly my cheeks } \\
\hline \multicolumn{6}{|l|}{ I feel very dry eyed } \\
\hline \multicolumn{6}{|c|}{ I have dry lips unless I use lip gloss on } \\
\hline \multicolumn{6}{|c|}{ I always drink water and tea to quench my thirst } \\
\hline \multicolumn{6}{|l|}{ I have dry cough with no sputum } \\
\hline \multicolumn{6}{|c|}{ My sputum is white and clear in color } \\
\hline Abdomen & & & & & \\
\hline I have constipation and hard sto & & & & & \\
\hline I feel unwell with cold food (e.g. & & & & & \\
\hline I have regular diarrhea & & & & & \\
\hline I wake up in the early morning $b$ & & & & & \\
\hline I feel tasteless in my mouth & & & & & \\
\hline I feel hungry even eating & & & & & \\
\hline I feel comfortable with hot water & & & & & \\
\hline I still feel thirsty even after havin & & & & & \\
\hline I urinate less frequently and it is & & & & & \\
\hline I urine frequently and it is clear & & & & & \\
\hline
\end{tabular}


(5) not suffering from any other chronic diseases or undergoing treatment like renal dialysis, chemotherapy, radiotherapy or under non-diabetic medications. Secondly, the study group was advised to (1) record a 7-day food intake either with photographs or written, (2) TCM Food Table was devised based on the collected food types from the food records, (3) perform a blood glucose test on 3 consecutive days, and (4) complete a demographic and health characteristics questionnaire. Thirdly, a registered TCM practitioner with over ten years of clinical experience assessed BC on each participant within one to two days after recording the food intake, using the Four Methods of TCM Diagnostic Technique. This involved collecting BCPs for guiding diagnosis of BC type (BCT). She analyzed participants' BCTs from head to toe following the Eight Principles of Syndrome Differentiation of Yin vs. Yang, interior vs. exterior, cold vs. heat, and deficiency vs. excess [47]. Finally, a clinic nurse took readings of the body mass index (BMI) of each participant. The second stage of research was a descriptive correlation study.

\section{Descriptive Correlation Study}

Prior to the second stage of research, three assessment questionnaires were developed. Results from the case study, validated questionnaires of Yindeficiency [37] and Yang-deficiency [38], and relevant TCM references [39-40] were used in developing the Yin/Yang-deficiency questionnaire (YY-DQ) (Table 1); and the food intake questionnaire (FI-Q) from the case study findings (Table 2). Firstly, the YY-DQ consists of 32 presentation items (YIDP: 19 items; YADP: 13 items), each score by the patient on a scale from 0 (never) to 1 (rare), 2 (occasionally), 3 (often), and 4 (always). A higher score indicated more frequent presentation. The YY-DQ was categorized by presentation scores into four levels using median as the cut point. As for YIDPs, $0-10$ scores as low presentation; 11-20 scores as moderate presentation; 21-30 scores as high presentation; and $>30$ scores as very high presentation. For YADPs, 0-8 scores as low presentation; 9-15 scores as moderate presentation; 16-34 scores as high presentation; and $>34$ scores as very high presentation. Content validity was established by three registered TCM practitioners of whom one obtained university degree in Chinese medicine and has clinical experience for more than 25 years. The other two TCM practitioners have been practising TCM for eight and 18 years respectively.

Secondly, response scales for 15 hot/warm and 15 cold/cool food items were based on frequency of food intake over seven days prior to the survey. With reference to the results from phase-one study, consumption of hot/warm and cold/cool foods for a minimum of three times in seven days can cause cold and hot natures of BCPs respectively. Based on this empirical finding, we set each score on a scale from 0 (0 frequency) to 1 (1-2 times), 2 (3-4 times), and 3 (more than 4 times). A higher score indicates more frequent intake. The FI-Q was categorized into intake score by four levels using median as the cut point. For food intake in hot/warm nature, 0-7 scores as low intake; 8-13 scores as moderate intake; $14-29$ scores as high intake; and $>29$ scores as very high intake. For food intake in cold/cool nature, 0-8 scores as low intake; 9-15 scores as moderate intake; $15-30$ scores as high intake; and $>30$ scores as very high intake. Content validity was established by (a) one herbalist having a university degree in herbal medicine and has been practising in this field for more than five years and (b) two registered TCM practitioners who have university degrees and have at least 25 years of clinical experience in TCM. Face validity was established for both $Y Y-D Q$ and FI-Q by having ten T2DM patients at a DM outpatient clinic who were recruited on a voluntary basis. This was followed by a pre-test with an interval of 2 weeks. The participants in the pre-test included five T2DM patients from a non-profit association for persons with DM. Apart from the YY-DQ and FI-Q, a demographic and health characteristics questionnaire was adapted from the one used in the case study. Two questions were added including "Do you have other chronic disease apart from DM?' and 'Are you on nonDM medication?" These two questions served to seek if other chronic diseases and non-DM medications were contributory factors to BCPs from a TCMP [41-42].

A sample of 104 and 70 persons with T2DM and non-DM respectively was recruited from a program of World Diabetes Day 2013 that was held in the Hong Kong Polytechnic University. The sample also included two health rehabilitation networks, and one self-help society for persons with DM. At the survey, each participant completed the YY-DQ, FI-Q, and demographic and health characteristics questionnaire followed by data verification conducted by the researcher or trained helpers.

\section{Data Analysis}

\section{Case Study}

In phase one, case study approach was employed to examine the BCPs, BCT, food intake, and demographic and health characteristics of each 
Table 2: The Food Intake Questionnaire (FI-Q)

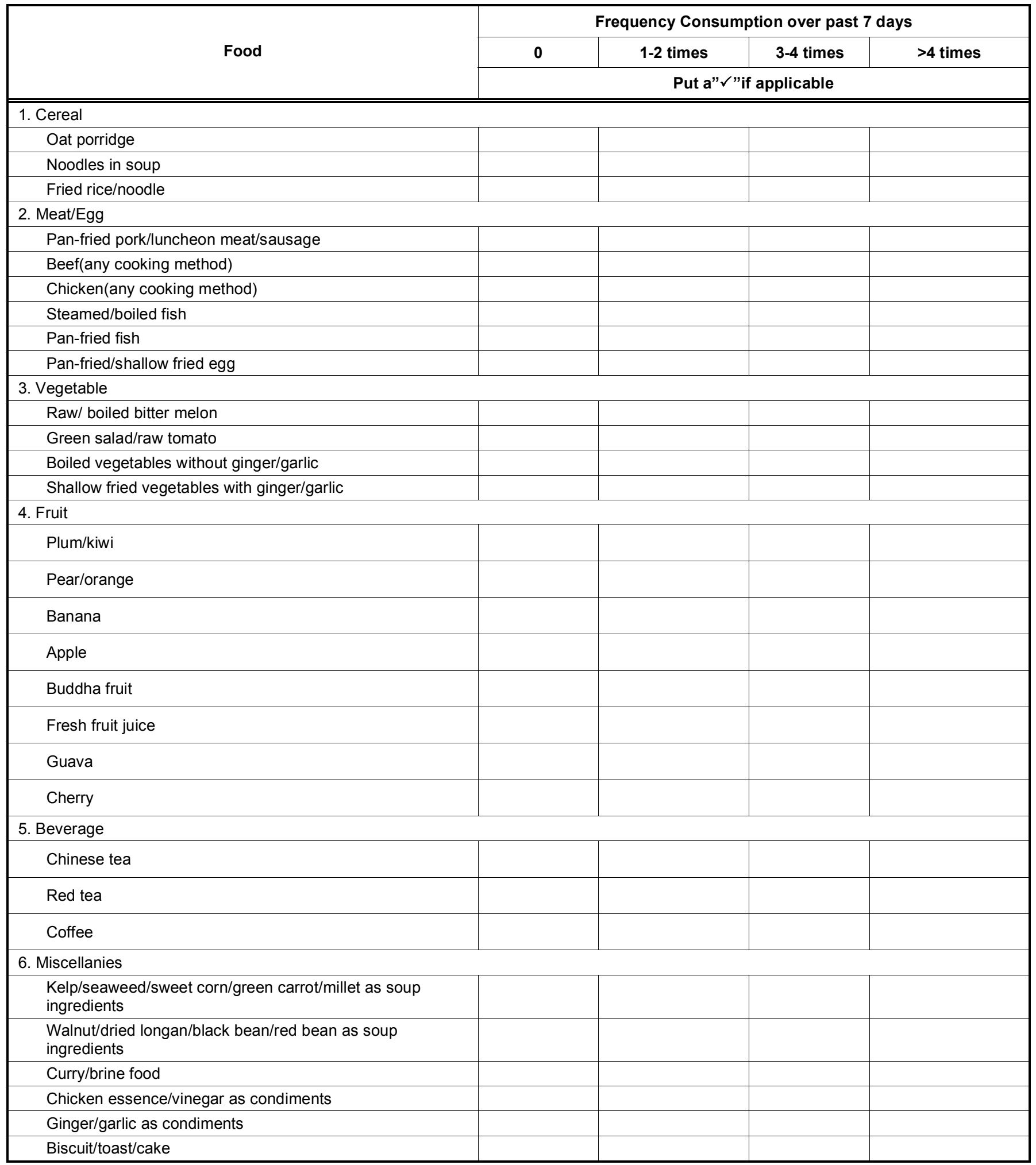

participant (Figure 1). A case was defined as a participant and its units of analysis were defined as demographic and health characteristics, BCPs, BCTs, food intake by TCM food natures, and correlation between the food intake in TCM food natures and
$\mathrm{BCPs}$ and BCT. Case-base and cross-case analyses were performed to examine BCPs, BCTs, food intakes in TCM food natures, and demographic and health characteristics of different cases and similar BC groups respectively. 


\section{Descriptive Correlation Study - Statistical Analysis}

The Statistical Package for Social Sciences (SPSS, version 20; SPSS Inc., USA) was used to analyze the questionnaire data in phase-two study. Descriptive analysis was performed by (1) percentage of frequency for demographic and health characteristics, (2) ANOVA for comparing YIDPs, YYDPs, and YYDPs in the samples of the T2DM and non-DM, (3) median as cut point for defining presentation scores for $Y Y-D Q$ and food intake scores for $\mathrm{FI}-\mathrm{Q}$, and (4) mean \pm SD for scores of YIDPs, YADPs, and food intakes in hot/warm and cold/cool natures. Due to a non-normally distributed data set, Spearman's correlation coefficient was used to examine correlations between YIDPs, YADPs, YYDPs and food intakes in hot/warm and cold/cool natures; and correlations between YIDPs, YADPs, YYDPs and food types in TCM. $P<0.05$ was considered statistically significant.

\section{Scientific Rigor}

In phase-one study, the researcher verified collected data by face-to-face interview with individual participants and telephoning with the TCM practitioner and nurse. The TCM Food Table was categorized from 223 collected food types with references to eight TCM food and diet books selected systematically from over 100 relevant references and a TCM panel discussion. Inter-rater reliability tests were performed to validate the assessed BC status and nutritional values of food intakes. Agreement between the investigators and the inter-raters was $80 \%$. The participants were asked to contact the researcher afterwards, if they remembered anything they wanted to add and five participants did. To maintain the reliability, the participants, TCM practitioner, clinic nurse, and instruments remained unchanged during the anthropometric and clinical measurements including body weight and height for the calculation of BMI and blood glucose tests, throughout the study period. Methodological triangulation of the data sets [43] was used to compare the results of correlations between BCPs and food intake in TCM from quantitative analysis in phase-two study.

\section{RESULTS}

\section{Participants' Demographic and Health Characteri- stics}

Of the 18 cases in phase-one study, female participants and those aged below 60 were in the majority (see Table 3). Over $60 \%$ had longer years of
Table 3: Demographics and Health Characteristics of Case Study $(n=18)$

\begin{tabular}{|c|c|}
\hline Characteristics $(n=18)$ & $n(\%)$ \\
\hline \multicolumn{2}{|l|}{ Gender } \\
\hline Female & $14(77.8)$ \\
\hline Male & $4(22.2)$ \\
\hline \multicolumn{2}{|l|}{ Age } \\
\hline 30-39 & $3(16.7)$ \\
\hline $40-49$ & $7(44.4)$ \\
\hline $50-59$ & 6(33.3) \\
\hline $60-70$ & $1(5.50)$ \\
\hline$>70$ & $2(11.1)$ \\
\hline \multicolumn{2}{|l|}{ Education } \\
\hline Primary school & $1(5.60)$ \\
\hline Secondary school & $9(50.0)$ \\
\hline Tertiary & $8(44.4)$ \\
\hline \multicolumn{2}{|l|}{ Occupation } \\
\hline Business owner & $1(5.60)$ \\
\hline Professional & $1(5.60)$ \\
\hline Clerical support & $8(44.4)$ \\
\hline Housewife & $3(16.6)$ \\
\hline Retired & $3(16.6)$ \\
\hline Unemployed & $2(11.1)$ \\
\hline \multicolumn{2}{|l|}{ Diabetes family history } \\
\hline Yes & $11(61.1)$ \\
\hline No & $5(27.8)$ \\
\hline Unknown & $2(11.1)$ \\
\hline \multicolumn{2}{|l|}{ Years of known diagnosis } \\
\hline$<6$ months & $1(5.60)$ \\
\hline 6 months -1 year & $1(5.60)$ \\
\hline $1-3$ years & $6(33.3)$ \\
\hline $3-5$ years & $4(22.3)$ \\
\hline $5-10$ years & $3(16.6)$ \\
\hline$>10$ years & $3(16.6)$ \\
\hline \multicolumn{2}{|l|}{ Body mass index } \\
\hline$<18.5$ (underweight) & $1(5.50)$ \\
\hline 18.5-22.9 (normal weight) & $7(38.9)$ \\
\hline 23-24.9 (overweight) & $4(22.2)$ \\
\hline 25-29.9 (very overweight) & $3(16.7)$ \\
\hline$>30$ (obese) & $3(16.7)$ \\
\hline \multicolumn{2}{|l|}{ Diabetic treatment } \\
\hline OHA (oral glycaemia agent, diet) & $2(11.1)$ \\
\hline OHA, diet, exercise & $14(77.8)$ \\
\hline Diet, exercise & $2(11.1)$ \\
\hline \multicolumn{2}{|l|}{ Regular aerobic exercise } \\
\hline Yes & $16(88.9)$ \\
\hline No & $2(11.1)$ \\
\hline \multicolumn{2}{|l|}{ Blood glucose control } \\
\hline Satisfactory & $16(88.9)$ \\
\hline Unsatisfactory & $2(11.1)$ \\
\hline
\end{tabular}

known diagnosis ( $>5$ years) while overweight $(\mathrm{BMI}>$ 23) was found in more than half of the sample. Nearly 
all the participants were treated with oral hypoglycaemia agent $(\mathrm{OHA})$ and their blood glucose levels were under good control. In phase-two study, survey participants were classified into T2DM and nonDM groups (see Table 4). Since there were no

Table 4: Demographic Characteristics of the Descriptive Correlation Study $(n=210)$

\begin{tabular}{|c|c|}
\hline & Frequency (\%) \\
\hline \multicolumn{2}{|l|}{ Gender } \\
\hline Male & $57(27.1)$ \\
\hline Female & $153(72.9)$ \\
\hline \multicolumn{2}{|l|}{ Age (years old) } \\
\hline Below 60 & $84(40.4)$ \\
\hline Over 60 & $124(59.6)$ \\
\hline \multicolumn{2}{|l|}{ Education level } \\
\hline Primary or below & $108(51.2)$ \\
\hline Secondary & $80(37.9)$ \\
\hline Tertiary & $20(9.50)$ \\
\hline \multicolumn{2}{|l|}{ Occupation } \\
\hline Manual worker & $17(8.10)$ \\
\hline Clerical & $19(9.00)$ \\
\hline Professional & $6(2.80)$ \\
\hline Housewife & $78(37.0)$ \\
\hline Unemployed & $1(0.50)$ \\
\hline Retired & $83(39.3)$ \\
\hline Student & $6(2.80)$ \\
\hline \multicolumn{2}{|l|}{ Shift Work } \\
\hline Yes & $4(1.90)$ \\
\hline No & $37(17.5)$ \\
\hline Not applicable & $69(80.1)$ \\
\hline \multicolumn{2}{|l|}{ Home cooking } \\
\hline Self & $36(64.5)$ \\
\hline Family member & $58(27.5)$ \\
\hline Maid & $8(3.80)$ \\
\hline Rare home cooking & $4(1.90)$ \\
\hline \multicolumn{2}{|l|}{ Smoker } \\
\hline Yes & $7(3.30)$ \\
\hline No & 188(89.0) \\
\hline Ex-smoker & $15(7.10)$ \\
\hline \multicolumn{2}{|l|}{ Drinker } \\
\hline Yes & $7(3.30)$ \\
\hline No & 192(91.0) \\
\hline Ex-drinker & $10(4.10)$ \\
\hline \multicolumn{2}{|c|}{ Verification of completed questionnaires } \\
\hline Without verification & $9(4.30)$ \\
\hline With verification & 201(95.7) \\
\hline
\end{tabular}

differences of YIDPs, YADPs, and YYDPs between these groups (YIDPs: T2DM $=19.4 \pm 9.8$, non$\mathrm{DM}=19.6 \pm 9.8, p=0.93$; YADPs: $\mathrm{T} 2 \mathrm{DM}=19.4 \pm 9.8$, non$\mathrm{DM}=14.7 \pm 7.7 p=0.74$; YYDPs: $\mathrm{T} 2 \mathrm{DM}=34.1 \pm 17.2$, non$D M=34.6 \pm 16.2, p=0.83$ ), a sample of 210 participants was used for analysis in the descriptive correlation study. Female was in a majority while less than half of the survey sample was aged below 60 (see Table 4). The mean age was $62.6 \pm 12.9$. Similar to phase-one study, longer years of known diagnosis ( $>5$ years) was in a majority (see Table 5). About $50 \%$ were found to be overweight $(\mathrm{BMI}>23)$. The mean body weight $(\mathrm{kg})$ and $\mathrm{BMI}$ were $59.3 \pm 11.8$ and $23.6 \pm 4.1$ respectively. About half of the respondents were treated with $\mathrm{OHA}$. Upon the survey, immediate blood glucose test results showed that over $70 \%$ of the participants' blood glucose levels were within normal range (see Table 5). Regarding to the question on "other chronic disease", about half of the survey participants responded "yes". Among the reported chronic diseases, about $65 \%$ were hypertension. Only about one-third of the survey participants were prescribed with non-DM medications.

\section{BCPs}

In phase-one study, a total of 28 BCPs were identified. YADP (cold nature) was prevalent in the majority $(n=17)$ while the $n$-value for YIDP (hot nature) was just 11 . Of the 18 cases, $B C$ types were classified as follows: YID in three cases, YAD in 12 cases, and YYD in three cases. In phase-two study, moderate presentation scores were shown both in YIDPs $($ mean $\pm S D=19.5 \pm 7.5)$ and YADPs $(m e a n \pm S D=$ $14.8 \pm 7.9)$.

\section{Food Intake in TCM}

223 food types were compiled from phase-one study. Disregard of the food types of neutral nature (79, $35.4 \%$ ), food types of cold/cool nature comprised a majority $(77,34.5 \%)$ than those of hot/warm nature $(67$, $30.1 \%)$. In phase-two study, moderate intake score was found both in the food intake in hot/warm nature (mean $\pm S D=13.4 \pm 5.7)$ and cold/cool nature (mean $\pm S D$ $=14 \cdot 6 \pm 5 \cdot 2)$.

\section{Correlation between BC and Food Intake in TCM}

In phase-one study, two of the three YID cases had food intake higher in hot/warm nature; 11 of the 12 YAD cases had food intake higher in cold/cool nature; and two cases and one case with YYD had food intake in extremely high or higher in cold/cool nature 
Table 5: Health Characteristics of the Descriptive Correlation Study $(n=210)$

\begin{tabular}{|c|c|}
\hline & Frequency (\%) \\
\hline \multicolumn{2}{|l|}{ DM Status } \\
\hline Type 2 DM & $140(66.7)$ \\
\hline Non-DM & $70(33.3)$ \\
\hline \multicolumn{2}{|c|}{ Years of known diagnosis } \\
\hline$<1$ year & $25(11.8)$ \\
\hline $1-3$ years & $22(10.4)$ \\
\hline $3-5$ ears & $4(6.60)$ \\
\hline $5-10$ years & $24(11.4)$ \\
\hline$>10$ years & $53(25.1)$ \\
\hline Not applicable & $70(33.2)$ \\
\hline \multicolumn{2}{|l|}{ Oral hypolycaemia agent } \\
\hline Yes & $103(51.2)$ \\
\hline No & $98(48.8)$ \\
\hline \multicolumn{2}{|l|}{ Non-DMdisease } \\
\hline Yes & $105(51.2)$ \\
\hline No & $100(48.5)$ \\
\hline \multicolumn{2}{|l|}{ Non-DMmedication } \\
\hline Yes & $77(37.4)$ \\
\hline No & $127(61.7)$ \\
\hline Non-DM disease types & $105(51.2)$ \\
\hline Hypertension & $70(66.7)$ \\
\hline Heart disease & $11(10.6)$ \\
\hline Hyperlipidemia & $7(6.7)$ \\
\hline Hay fever & $4(3.9)$ \\
\hline Cancer uterus & $2(2.0)$ \\
\hline Cancer gall bladder & $2(2.0)$ \\
\hline Gout & $1(0.9)$ \\
\hline Liver disease & $1(0.9)$ \\
\hline Rheumatic arithritis & $1(0.9)$ \\
\hline Asthma & $1(0.9)$ \\
\hline Chronic bronchitis & $1(0.9)$ \\
\hline Pancreatitis & $1(0.9)$ \\
\hline Chronic pain & $1(0.9)$ \\
\hline Mental disorder & $1(0.9)$ \\
\hline Transplanted kidney & $1(0.9)$ \\
\hline \multicolumn{2}{|c|}{ Aerobic exercise $\geq 3$ times/week } \\
\hline Yes & $153(74.6)$ \\
\hline No & $51(24.9)$ \\
\hline \multicolumn{2}{|l|}{ Blood glucose control } \\
\hline Below normal & $(1.8)$ \\
\hline Normal & $123(72.8)$ \\
\hline Above normal & $40(23.7)$ \\
\hline \multicolumn{2}{|l|}{ Body mass index (BMI) } \\
\hline Below 23 & $88(41.7)$ \\
\hline Above 23 & $99(52.9)$ \\
\hline
\end{tabular}

respectively. In phase-two study, YIDPs, YAPDs, and YYDPs highly correlated with the food intake in hot/warm nature and cold/cool (see Table 6). The results also showed that YIDPs highly correlated with female participants (YIDPs: female $=20.4 \pm 9.8$, male $=17.1 \pm 10.3, p=0.037)$; BCPs highly correlated with age $\quad<60$ (YIDPs: age $<60=22.2 \pm 9.2$, age $>60=17.5 \pm 10.1, \quad p=0.001 ; \quad$ YADPs: age $<60=15.4 \pm 6.6, \quad$ age $>60=14.3 \pm 8.7, \quad p=0.035$; YYDPs: age $<60=37.6 \pm 14.4$, age $>60=31.8 \pm 17.4$, $p=0.012$ ); and non-DM chronic diseases also showed positive correlations with YIDPs, YADPs, and YYDPs (YIDPs: $y e s=20.9 \pm 9.8, \quad$ no $=17.9 \pm 10.1, \quad p=0.036$; YADPs: $y e s=16.2 \pm 8.0$, no $=13.3 \pm 8.0, p=0.036$; YYDPs: yes $=37.0 \pm 15.8$, no=31.2 $\pm 16.8, p=0.011)$. The study findings showed that four food types of hot/warm nature closely correlated with YID type of $B C$ and six YIDP items while four food types of cold/cool nature closely correlated with YAD and/or YYD types of BC, three YADPs, and one YIDP item (see Table 7).

\section{DISCUSSION}

\section{Classification of BC in DM}

In this mixed method study, YIDPs, YADPs, and YYDPs were identified. According to the diagnostics of TCM which is a study concerning the examination of body, diagnosis of diseases and classification of BCTs under the guidance of the basic theories of TCM [44], persons with majority of YIDPs or YADPs were assessed to have YID or YAD type of $B C$ respectively. For those who have equal or nearly equal YIDPs and YADPs are diagnosed having YYD type of $B C$. In this connection, YID, YAD, and YYD types of $B C$ were found in this study. The results are consistent with TCM references that YID [18-19], YAD [16-17], and YYD [19] are common BC types in DM population. Comparatively speaking, YIDP as analyzed from phase-two study was in the majority. This is in agreement with Si \& Li [45] that YID more prevalent in DM followed by YAD and YYD. Surveys reported that $14.8 \%$ to $31.9 \%$ of the healthy populations were identified to have YID [46]. YID type of BC in healthy population can be considered to be one kind of "subhealth" (sub-optimal health). "Sub-health" is defined to be the health status of a person between healthy and unhealthy stage [47]. In fact, YID type of BC is one kinds of "pathological" BCs which is developed from "unhealthy" BC, such as "Cold-dampness", "Dampnessheat", and "Cold- or Heat-dryness" with a subnormal body temperature and humidity and clinical presentations such as malaise, insomnia, and overweight [8]. If persons with a YID type of BC can be assessed immediately and provided appropriate treatment before DM occurs, one can mitigate the 
Table 6: Spearman's Correlations of Yin-deficiency, Yang-deficiency, Yin-Yang-deficiency Presentations and Food Intakes in TCM $(n=210)$

\begin{tabular}{|c|c|c|c|}
\hline \multirow{2}{*}{ Food intake in TCM } & \multicolumn{3}{|c|}{ Spearman's rho ( $p$-value) } \\
\hline & \multirow{3}{*}{$\begin{array}{c}\text { YIPDs } \\
0.34(p=0.000) \\
0.18(p=0.006)\end{array}$} & \multirow{3}{*}{$\begin{array}{c}\text { YADPs } \\
0.18(p=0.008) \\
0.20(p=0.006)\end{array}$} & \multirow{3}{*}{$\begin{array}{c}\text { YYDPs } \\
0.29(p=0.006) \\
0.20(p=0.003)\end{array}$} \\
\hline Hot/warm foods & & & \\
\hline Cold/cool foods & & & \\
\hline \multicolumn{4}{|l|}{ Hot/warm food types ( $n, \%)$} \\
\hline Fried rice/noodle $(140,66.7)$ & $0.09(p=0.21)$ & $-0.40(p=0.57)$ & $0.31(p=0.65)$ \\
\hline Fried pork/luncheon meat/sausage $(108,51.4)$ & $0.21(p=0.002)$ & $0.48(p=0.49)$ & $0.13(p=0.64)$ \\
\hline Beef (all cooking methods) $(100,47.6)$ & $0.11(p=0.09)$ & $0.15(p=0.50)$ & $0.14(p=0.46)$ \\
\hline Chicken (all cooking methods) $(168,80.5)$ & $0.16(p=0.02)$ & $0.13(p=0.07)$ & $0.14(p=0.04)$ \\
\hline Pan-fries/deep-fried fish $(87,41.4)$ & $0.11(p=0.12)$ & $0.06(p=0.42)$ & $0.08(p=0.25)$ \\
\hline Pan-fried egg $(139,66.2)$ & $0.18(p=0.01)$ & $0.13(p=0.06)$ & $0.16(p=0.02)$ \\
\hline Shallow fried vegetables with ginger/garlic $(108,51.4)$ & $0.11(p=0.13)$ & $0.04(p=0.59)$ & $0.08(p=0.24)$ \\
\hline Guava $(58,27.6)$ & $-0.07(p=0.34)$ & $-0.03(p=0.64)$ & $-0.05(p=0.49)$ \\
\hline Cherry $(43,20.3)$ & $0.10(p=0.14)$ & $0.11(p=0.11)$ & $0.12(p=0.08)$ \\
\hline Coffee $(87,41.4)$ & $0.12(p=0.07)$ & $0.06(p=0.43)$ & $0.11(p=0.13)$ \\
\hline $\begin{array}{l}\text { Soup (walnut/dried-longan/black bean/red bean) } \\
(95,45.2)\end{array}$ & $0.21(p=0.000)$ & $0.18(p=0.01)$ & $0.21(p=0.003)$ \\
\hline Curry/brine $(67,31.9 \%)$ & $0.21(p=0.000)$ & $0.12(p=0.09)$ & $0.18(p=0.008)$ \\
\hline Chicken essence/vinegar as condiments $(113,53.8)$ & $0.22(p=0.001)$ & $0.11(p=0.12)$ & $0.15(p=0.030)$ \\
\hline Ginger/garlic/green onion as condiments $(165,78.5)$ & $0.29(p=0.000)$ & $0.14(p=0.05)$ & $0.21(p=0.003)$ \\
\hline Biscuit/toast/cake $(174,83.3)$ & $0.21(p=0.002)$ & $0.11(p=0.10)$ & $0.18(p=0.008)$ \\
\hline \multicolumn{4}{|l|}{ Cold/cool food types $(n, \%)$} \\
\hline Oat porridge $(145,69.0)$ & $-0.03(p=0.65)$ & $0.07(p=0.34)$ & $0.01(p=0.99)$ \\
\hline Noodle in soup $(171,81.4)$ & $0.14(p=0.03)$ & $0.10(p=0.34)$ & $0.13(p=0.06)$ \\
\hline Steamed/boiled fish $(174,83.3)$ & $0.01(p=0.87)$ & $0.06(p=0.42)$ & $0.04(p=0.59)$ \\
\hline Raw/steamed/boiled bitter melon (93, $44.3 \%)$ & $0.03(p=0.66)$ & $0.02(p=0.90)$ & $0.03(p=0.66)$ \\
\hline Green salad (raw vegetables) $(112,53.3)$ & $0.12(p=0.08)$ & $0.15(p=0.03)$ & $0.19(p=0.006)$ \\
\hline Boiled vegetables without ginger/garlic $(149,70.9)$ & $0.11(p=0.10)$ & $0.15(p=0.03)$ & $0.13(p=0.06)$ \\
\hline Plum/kiwi fruit $(67,31.9)$ & $0.04(p=0.60$ & $0.04(p=0.57)$ & $0.04(p=0.60)$ \\
\hline Pear/orange $(164,78.1)$ & $0.09(p=0.20)$ & $0.11(p=0.11)$ & $0.10(p=0.16)$ \\
\hline Banana $(128,60.9)$ & $0.11(p=0.10)$ & $0.06(p=0.43)$ & $0.08(p=0.24)$ \\
\hline Apple $(149,70.9)$ & $0.10(p=0.18)$ & $0.08(p=0.28)$ & $0.09(p=0.20)$ \\
\hline Buddha fruit $(36,17.1)$ & $0.10(p=0.17)$ & $0.11(p=0.11)$ & $0.10(p=0.14)$ \\
\hline Fresh fruit juice $(37,17.6)$ & $0.13(p=0.07)$ & $0.06(p=0.41)$ & $0.14(p=0.04)$ \\
\hline Chinese tea $(164,78.1)$ & $0.01(p=0.90)$ & $0.04(p=0.59)$ & $0.02(p=0.76)$ \\
\hline Red tea $(90,42.9)$ & $0.07(p=0.33)$ & $-0.05(p=0.48)$ & $0.03(p=0.72)$ \\
\hline $\begin{array}{l}\text { Kelp/seaweed/sweet corn/green carrot/millet as soup } \\
\text { ingredients }(148,70.5 \%)\end{array}$ & $0.20(p=0.003)$ & $0.17(p=0.001)$ & $0.21(p=0.002)$ \\
\hline
\end{tabular}

YIDPs=Yin-deficiency presentations; YADPs=Yang-deficiency presentations; YYDPs=Yin-Yang-deficiency presentations.

increasing rate of DM prevalence and other chronic diseases such as hypertension. In this connection, an early diagnosis of the YID type of BC can provide an effective intervention strategy for health or medical professionals to treat people with YID before the diseases occur.
In phase-two study, female participants with YIDP and aged over 60 years were comparatively more than the male respondents. It is said in the Yellow Emperor's Canon of Internal Medicine: "After the age of forty-nine, females are declining, her menstruation being exhausted. Her physique becomes old and 
Table 7: Spearman's Correlation Coefficient between Body Constitutions and Food Types in TCM

\begin{tabular}{|c|c|c|}
\hline Food type & Phase-one: case study & Phase-two: descriptive correlation study \\
\hline & Body constitution types & $\begin{array}{l}\text { Body constitution presentations } \\
\text { Spearman's rho ( } p \text {-value) }\end{array}$ \\
\hline $\begin{array}{l}\text { Deep fried/pan fried luncheon } \\
\text { meat/fish/egg }\end{array}$ & YID (Mr. D, Ms. I) & $\begin{array}{c}\text { YIDPs }(0.20, p=0.002) ; \text { I wear thin clothes due to fear of the hot } \\
\text { temperature }(0.21, p=0.002)\end{array}$ \\
\hline Curry beef bun & YID (Mr. D) & $\begin{array}{l}\text { YIDPs }(0.21, p=0.000) ; \text { I have hot flush at night }(0.26, p=0.000) \\
\text { I always drink water to quench my } \\
\text { thirst }(0.20, p=0.004) ; \text { I feel very dry eyes } \\
(0.20, p=0.005) \\
\text { YYDPs }(0.18, p=0.008) \text { - }\end{array}$ \\
\hline Biscuit/cake/pan cake/sponge cake & YID (Ms. I) & $\begin{array}{l}\text { YIDPs }(0.20, p=0.002) ; \text { I feel on and off hot and cold }(0.24 \\
\begin{array}{c}(p=0.001) ; \text { I always drink water to quench } \\
\text { my thirst }(0.19, p=0.006) ; \text { I have tinnitus } \\
(0.20, p=0.004) \\
\text { YYDPs }(0.18, p=0.008) \text { - }\end{array}\end{array}$ \\
\hline $\begin{array}{l}\text { Boiled vegetable without } \\
\text { ginger/garlic }\end{array}$ & $\begin{array}{l}\text { YAD (Mr. B, Ms. C, Ms. E, } \\
\text { Ms. G, Mr. H, Mr. J) }\end{array}$ & YADPs $(0.15, p=0.03) ;$ I feel unwell with wind blow $(0.21, p=0.003)$ \\
\hline Raw/boiled bitter melon & $\begin{array}{l}\text { YAD (Mr. B, Mr. J, Mr. N) } \\
\text { YYD (Ms. R) }\end{array}$ & nil I have long urination in clear colour $(0.25, p=0.002)$ \\
\hline Green salad (raw vegetable) & $\begin{array}{l}\text { YAD (Mr. B) } \\
\text { YYD (Ms. A) }\end{array}$ & $\begin{array}{c}\text { YADPs }(0.15, p=0.03) ; \text { I have regular diarrhea }(0.19, p=0.005) \\
\text { YYDPs }(0.19, p=0.006)\end{array}$ \\
\hline Orange juice & YYD (Ms. M) & YYDPs $(0.14, p=0.04) ;$ I have hot flush at night $(0.19, p=0.006)$ \\
\hline
\end{tabular}

YID=Yin-deficiency; YAD=Yang-deficiency; YYD=Yin-Yang-deficiency; YIDPs=Yin-deficiency presentations; YADPs=Yang-deficiency presentations; YYDPs=YinYang-deficiency presentations.

feeble, and by then, she can no more conceive" [15]. Females over forty-nine are prone to have YID due to the declination of her body Yin. Without regulation, the declination of body Yin will further progress to become YYD according to the theory of Yin-Yang dependence [17]. This was highly consistent with the findings from phase-two study (YYD: female=35.7 \pm 16.3 ; male $=30.4 \pm 16, \quad p=0.004)$. Earlier assessment and regulation of the YIDPs likely occur in women over 49 years might either prevent disease occurrence or YYD type of BC.

In phase-one study, all the three cases with YYD were below 60 years (Table 3 ). This is not consistent with Zhou et al's. [12] finding that YYD occurred in DM persons aged over 60. Results from phase-two study corresponded with those in phase one. YYDPs mostly occurred in persons with DM aged below 60 years (mean \pm SD: $<60$ years $=37.6 \pm 14.4 ;>60$ years $=$ 31.2 $\pm 16.8, p=0.011)$. In 2003-2004, the DM prevalence in Hong Kong among the older population aged 65 and above was six times compared to the younger population aged 18 to 64 [48]. It is fortunate that based on the findings from this study, YYD is not a necessary BC type in this older DM population. However, it occurs mostly in persons with DM aged below 60 years. Prevention measures should be introduced to persons with DM of all ages regardless of whether they have YID or YAD, to prevent the onset of YYD.

In phase-two study, it was shown that survey participants who had non-DM chronic diseases had more deviated BCPs than without. Of the fifteen nonDM chronic diseases found in the survey sample, hypertension was in the majority (see Table 5). YID was a common $B C$ type in persons with hypertension apart from $B C$ of $Y A D$, phlegm-dampness, and gentle nature [28]. If left unregulated, YID and YAD will persist in persons with hypertension. According to the theories of Yin-Yang's mutual transformation and Yin-Yang interdependence, an overly excessive Yang (YID) or Yin (YAD) can change into YAD or YID respectively or further weaken the opposite side, thus leading to YYD. 


\section{BC and Food Intake in TCM}

Of the three cases with YID in phase-one study, two had food intake higher in hot/warm nature. Results from phase-two study showed that YIDPs highly correlated with hot/warm food intakes (see Table 6). The results are consistent with the findings from phaseone study in which Mr. D had hot/warm foods such as deep-fried/pan-fried luncheon meat/fish/egg every day. This is evident from its high correlation with "I wear thin clothes due to the fear of hot temperature" (see Table 7). Tang et al. [49] reported that male infertility was associated with geographical, social and economical, cultural, and diet factors in Zhuang males having YID type of BC. They ate a lot of smoked meats and fish, and barbeque meats that were hot in nature causing depletion of body fluids leading to YID with heat and dryness. Hence, a prolonged intake of food of hot/warm nature can potentially have an impact on BC progressing to YID.

In phase-one study, food intake higher in cold/cool nature was found in all YAD cases except one case. The findings from phase-two study showed that YADPs highly correlated with food intake in cold/cool nature (see Table 6). Chao et al. [50] conducted a study on the effects of water (neutral nature), aged ginger tea (hot nature) and coconut water (cold nature) on signals of heart rate variability and nail fold microcirculation of 19 young persons with YID type of $\mathrm{BC}$ and 11 with $Y A D$ type of $B C$. The results showed that the skin temperature of the YID group after taking coconut water was significantly reduced compared to their skin temperature after taking water or aged ginger tea ($10.1 \%, p<0.05)$. The capillary red blood cell velocity in nail fold microcirculation of the YAD group accelerated significantly after taking the hot attribute aged ginger tea compared with taking water or coconut water $(+16.6 \%, p<0.05)$. The capillary red blood cell velocity in nail fold microcirculation of the YID group decelerated significantly after taking coconut water compared with taking water or aged ginger tea ($26.7 \%, p<0.05)$. Hence, YAD is influenced by food of $\mathrm{cold} / \mathrm{cool}$ nature but regulated by food of hot/warm nature. In this mixed method study, green salad (raw vegetables) and boiled vegetables without ginger/garlic were found to be correlated highly with YADPs including "I feel unwell with wind blowing" and "I have regular diarrhea" (see Table 7). Thus, these food types of cold/cool nature should be avoided for persons with YAD, especially when the temperature is generally low.

Phase-two study findings showed that YYDPs correlated highly with food intake in cold/cool nature (see Table 6). This is consistent with the phase-one study findings that all the three YYD cases had food intake higher in cold/cool nature especially two had extremely high intake. It is because "Yin becomes deficient while Yang is weakened and vice versa" [15] according to the theory of Yin-Yang interdependency. Deficiency of either Yin or Yang causes the weakening of both Yin and Yang [17]. In this mixed method study, orange juice (cold nature) highly correlated with "I have hot flush at night" (hot nature) in YYDPs (see Table 7). This is consistent with Ms. M of phase-one study where daily intake of orange juice caused her YYD type of BC. Frequent intake of fruit juice may not be advisable for consumption in persons with DM for its potential in influencing YYDPs apart from raising the blood glucose level with its high sugar content.

The phase-two study had found positive correlations between YIDPs and food intake higher in hot/warm nature, YADPs and food intake higher in cold/cool, and YYDPs with food intake higher in cold/cool nature. These results largely supported the phase-one study findings while they also suggested a third finding which highly revealed the mutual transformation between Yin and Yang. In TCM, different BCPs can be found in association with a single disease [51-52]. Likewise, the same BCP can be found to be associated with different diseases. In phase-two study, non-DM diseases were found positively correlated with YIDPs, YADPs, and YYDPs. In this case, survey participants with non-DM diseases might already have YIPDs, YADPs, or YYDPs. Persons with YIDPs due to non-DM diseases together with food intake higher in hot/warm nature as well as the generally low humidity in winter might result in an extremely excessive YID that might change to YAD presenting with YADPs in accordance with the Yin-Yang mutual transformation. Thus, food of hot/warm nature was found in persons with YADPs (see Table 6). Likewise, YADPs as a result of non-DM diseases together with food intake higher in cold/cool nature as well as the generally low temperature in winter might result in an extremely excessive YAD that might also change to YID with YIDPs. As a result, food of cold/cool nature was found in persons with YIDPs (see Table 6).

The findings of this mixed method study concluded that persons with T2DM and YIDPs, YADPs, or YYDPs tend to have food intakes higher in hot/warm nature or cold/cool nature.

\section{STUDY LIMITATIONS}

In phase-one study, we adopted a visualization technique to assess seven-day food intakes recorded 
by the 18 participants. The quantity and amount of these foods could only be assessed through estimation. Although $100 \%$ accuracy in food portioning might not be achievable by visualization, it did help in enhancing the accuracy of food assessment significantly. In phase-two study, we adopted the FI-Q to collect food frequency data. Since the food types in the FI-Q were selected from the TCM Food Table compiled between May and June in phase-one study where the temperatures were not generally low, some foods that the survey participants had consumed between October and December where the temperatures were generally low were not included in the FI-Q. Hot pot, mutton, and BBQ meats are foods commonly consumed by people during the winter when the temperatures are generally low. It is suggested that, to provide increased flexibility of food selection, specific foods or meals should be included in future studies in addition to the FI-Q. Besides, a comprehensive food FI-Q should also be developed for maximization of food selection.

\section{IMPLICATIONS FOR DIETETIC PRACTICE}

Correlation between BCPs and TCM food nature is supported in this mixed method study. Assessment of BCPs can be adopted in DM dietary therapy programs so that either Yin or Yang enhancing dietary therapy may be provided. Dieticians could learn about TCM, particularly, BC and TCM food theory so that regulations with respect to deviated BC could be applied. Patients with DM might be taught to self-identify their BCPs so that they could adjust their food intakes in different natures for either regulating respective BCPs or preventing inversely deviated BCPs.

$\mathrm{BC}$ assessment would be an important practice in community dietetics which could prevent chronic diseases such as DM and hypertension. It may be advisable to incorporate $\mathrm{BC}$ assessment into community health programs so that deviated BC could be identified earlier in persons with T2DM, who could receive Yin/Yang enhancing dietary program as soon as possible. Other potential community settings include rehabilitation centers, elderly centers, and women health centers etc.

\section{SUGGESTIONS FOR FUTURE STUDY}

Future study will be conducted to comprehend the association between BCP and daily food intake in persons with T2DM from a TCMP. It is necessary to test for factor validity of the $Y Y-D Q$ prior to an association study.

\section{ACKNOWLEDGEMENTS}

The authors want to thank the diabetes and health organizations for their supports in the recruitments. We wish to thank the organization committee of The World Diabetes Day 2013 (venue: The Hong Kong Polytechnic University), The Society for Wong Tai Sin Diabetes Mutual Help, The Hong Kong Society for Rehabilitation New Territories West Tai Hing Centre, The Hong Kong Society for Rehabilitation New Territories Prince of Wales Centre, and The Angel of Diabetic.

\section{REFERENCES}

[1] WHO. Country and regional data on diabetes. [Internet] 2014 [cited 2014 Apr 27]. Available from http://www.who.int/ diabetes/facts/world_figures/en/

[2] WHO. Diabetes program. [Internet]. 2009 [cited 2012 Nov 23]. Available from http://www.who.int/diabetes/en/

[3] Kuang DY. New version of human body constitution. J of Chin Med 2003; 21(4): 487-8 [in Chinese]

[4] Zhou TH, Wang HF, To CH, Suen KM, To, KL, Lee, Y, Muk, SK, Lo S, Wan, Y, Wang YN, et al. 217 diabetes cases in Chinese body constitutions and related causes. Beijing $\mathrm{J}$ TCM 2009; 28(1): 39-40 [in Chinese].

[5] Wang Q. The study progress and future of Chinese constitutionology. J of Shandong Uni Trad Chin Med 1994; 18 [in Chinese]

[6] Kuang QY. Outline of the constitutional food adjusting theory. J Zhejiang Chin Med Uni 2006; 30(3), 217-9 [in Chinese].

[7] Han YM, Zhang LT. Characteristic of constitution theory in traditional Chinese medicine. Zhongui Linchung Kanfu 2005 9: 130-1 [in Chinese].

[8] Chan YP, Chien WT. Concepts of body constitution, health and sub-health from traditional Chinese medicine perspective. World J Trans Med, 2(3): 56-64.

[9] Han YW, Zhou SN. Physical analysis of traditional Chinese medicine. Guiding J TCM 2010; 16(3): 3-5 [in Chinese].

[10] Quo Y, Cheun T. Progress of the study on the insulin resistance in type 2 diabetes. Inner Mongolia Chin Med 2006; (6): 50-2 [in Chinese].

[11] Yu PL, Wang KS. The cause of death in Chinese old people 1998, 1999, 2000. Chin J Epidemiol 2003; 24(1): 75 [in Chinese].

[12] Zhou TH, Wang HF, To $\mathrm{CH}$, Suen KM, To KL, Lee $\mathrm{Y}$, Muk SK., Lo S, Wang Y, Wang YN, et al. 217 diabetes cases in Chinese body constitutions and related causes; Beijing $\mathrm{J}$ TCM 2009; 28(1): 39-40 [in Chinese].

[13] Qiao H.Z, Xing HS, Xu H, Zhang LH, Ning Q, Quang WT, Gui $Y Z$, Cai YF, et al. An investigation on the traditional Chinese medicine constitution of 1232 patients with slight or moderate primary hypertension in Lianyungang area. Chin J Basic Med in TCM 2011; 17(12): 1378-80 [in Chinese].

[14] Liu YX, Piao CL. Discussion on the balance of Yin and Yang theory of diabetes. Chin Med and Pharm 2012; 2(24): 98-9 [in Chinese].

[15] Wang B. (Tang dynasty). Yellow Emperor's Canon of Internal Medicine Chinese-English Edition. 2nd ed. In: Wu NL, Wu $A Q$, English translators. Beijing: China Science \& Technology Press 2010; p. 9-687.

[16] Suen KY. The concept model of Yin-qi and Yang-qi. J Chin Pharm 2005; 20(11) [in Chinese]. 
[17] Zuo YH (editor). Basic theory of traditional Chinese medicine. 5th ed. Shanghai: Shanghai Pujiang Education Press 2011; p. 14-123.

[18] Ao NG. The substance manifestations of Yin and Yang in traditional Chinese medical science. The Trad Asian Pacific Med 2007; 2:29-34 [in Chinese].

[19] Wang Q. Body Constitution in Chinese Medicine. 1st ed. Beijiang: China Pharmaceuticals Publisher 1995 [in Chinese]

[20] Zhu P, Lung SS. Analysis of the etiology and pathology of diabetes. J of Liaoning Uni TCM 2007; 11(6): 15-6 [in Chinese].

[21] Mau KW, Tsui K. New exploration of the diagnosis of Type 2 Diabetes. Study J TCM 2001; 19(3): 240-2 [in Chinese].

[22] Sun Simiao (Tang dynasty). Essential Prescriptions Worth a Thousand Gold for Emergencies. In: Muk I, editor. China: People of Jilin Publishers 1994 [in Chinese].

[23] Suen KY. The foundation of TCM theory. Beijiang: China Pharmaceuticals Publisher; 2002 [in Chinese].

[24] Chen B. The syndrome characteristics of senile diabetes. J Pract Trad Chin Inter Med 2012; 26(12):52-3 [in Chinese].

[25] Zhou TH, Wang HF, To CH, Suen KM, To KL, Lee $Y$, Muk SK, Lo S, Wang Y, Wang YN, et al.. 217 diabetes cases in Chinese body constitutions and related causes. Beijing Journal of TCM 2009; 28(1) [in Chinese].

[26] Yu CW, Cheung TF, Yeung LF, Wang YC. Survey of symptoms and research on body constitution in diabetes mellitus. J Shandong Uni TCM 2001; 25(3): 185-188 [in Chinese].

[27] Kuang DY. Food Therapy for Body Constitution and Healthy Life. 1st ed. Hong Kong: Commercial Press 2013; p. 3-4 [in Chinese].

[28] Shen CZ, Pang MC, Kwong HR, Cheng ZQ. The effect of Chinese food therapy on community dwelling Chinese hypertensive patients with yin-deficiency. J Clinl Nurs 2010; 19:1008-20.

[29] Ngai SM, Kam KL. Chinese food therapy. Beijiang: Chinese Medicine Pharmaceuticals Publisher; 2004 [in Chinese].

[30] Yung WK. Chinese Dietary Therapy. 2nd ed. Tien Tei Publisher; 1994 [in Chinese].

[31] Law MPM, Chuh AAT, Molinarit N, Lee A. An investigation of the association between diet and occurrence of acne: a rational approach from a TCM perspective. Clin Experi Dermat 2010; 35: 31-5.

[32] Lam SP. Dietary therapy for body constitution as preventive measure for children with infected respiratory system. Gi Lam Chin Med Pharma 2007; 27(7): 18-9.

[33] WHO. Ten facts about diabetes. [Internet]. 2014 [2014 Apr 27]. Available from http://www.who.int/features/factfiles/ diabetes/facts/en/index4.html

[34] Creswell J. Research design: qualitative, quantitative, and mixed methods approaches. Thousand Oaks 2003. CA: Sage.

[35] Yin RK. Case study research: design and methods. Thousand Oaks 2003. CA: sage.
[36] Salaris S. Meaning of the term-descriptive survey research methods. Inter J Trans in Business Management 2012; 1(6).

[37] Lee SJ, Park JH, Lee HS, Kim KH. Development and validation of Yin-deficiency questionnaire. The Am J Chin Med 2007; 15(1): 11-20.

[38] Wang HL, Kuo SH, Chou FH, Chen LM, Chen LL, Lin WT, et al. Reliability and validity evaluation of the traditional Chinese medicine Yang-Xu constitutional questionnaire on pregnant women. J Nurs and Healthcare Res 2012; 9(1): 3-11 [in Chinese].

[39] Shen CZ. The effect of Chinese food therapy on community dwelling middle-aged hypertensive patients with Yindeficiency in Hangzhou, China. The Hong Kong Polytechnic University 2009; p. 159 [Thesis in Chinese].

[40] Wang Q. Understanding body constitution for health and fitness. 1st ed. The Human Books Publication 2013; p. 62, 104 [in Chinese].

[41] Yu, CW, Zhang TF, Yang LF, Wang YC. Survey of symptoms and research on body constitution in diabetes mellitus. $J$ Shandong Uni TCM 2001; 25(3): 185-8 [in Chinese].

[42] Zhou HX, Jia HH, Zhou KW, Yuan JJ. Probing into the pathogenesis and syndrome of the diabetes in TCM. Chin J Medical Guide 2012; 14(3): 456-7 [in Chinese].

[43] Creswell JW. Research design: qualitative approaches London: Sage; 1994

[44] Beijing University of TCM. Diagnostics of Traditional Chinese Medicine, English-Chinese Collegiate Textbooks in TCM for Institutions of TCM of Higher Learning. Beijing: University of Beijing; 1998.

[45] Si FC, Li YN. Chinese constitution distribution in diabetes and drug regulation distribution. TCM Res 2009; 22(8) [in Chinese].

[46] Cheung M, Tai HY, Liu Y, Tung HW, Sze WH. Analysis of 1763 cases in community body constitution of Chinese medicine. Henan Chin Med 2002; 22(4): 71-3 [in Chinese].

[47] Wu MC, Chu WF, Li MC, Fan CK. Analysis of the Chinese medicine syndrome in partly Beijing people. Beijing Chin Med 2007; 26(6): 330-3 [in Chinese].

[48] McGhee SM, Ho LM, Cheung AW, Abdullah AS, Chan CW, Yeung AC. Evaluation of a mobile clinic for older people in Shamshuipo. Hong Kong Medical J 2007; 13(suppl 1): S135.

[49] Tang NG, Huang GH, Huang XF, Liang $\mathrm{CH}$. Discussion on male infertility in Zhuang population based on the theory of Chinese medical constitution. China Acad J Elect Publishing House 2012; 27(3) [in Chinese].

[50] Chao DP, Chen JJ, Huang SY, Tyan CC, Hsieh CL, Sheen LY. Effects of hot and cold foods on signals of heart rate variability and nail fold microcirculation of health young humans: a pilot study. Chin J Physio 2011; 54(3): 145-52.

[51] Ting SW, Ting M. Discussion on the clinical application of constitutionology. Jiangxi TCM 2003; 8:12 [in Chinese].

[52] Wang Q, Li YQ. Understanding and assessment of syndrome in Chinese medicine. Zhejiang J Chin Med 2006; 41(11): 623-5 [in Chinese].

\section{DOI: http://dx.doi.org/10.6000/1929-5634.2014.03.02.5}

(c) 2014 Wong et al.; Licensee Lifescience Global.

This is an open access article licensed under the terms of the Creative Commons Attribution Non-Commercial License (http://creativecommons.org/licenses/by-nc/3.0/) which permits unrestricted, non-commercial use, distribution and reproduction in any medium, provided the work is properly cited. 